\title{
A review on fuzzy control charts for monitoring attribute data
}

\begin{abstract}
Up to now, several methods have been proposed for monitoring processes with attribute data. These methods can be categorized into two major group; statistical methods and fuzzy methods. In this paper current fuzzy methods are introduced and the performance of fuzzy methods and statistical methods are compared together based on the Average Run Length (ARL). The comparison shows that the statistical method has the best performance. We show the necessity of using fuzzy method in case of attribute data. Then the critiques towards fuzzy methods are reviewed which show the usage of fuzzy set theory in these methods have some restriction. As a result we indicate a study gap about the usage of fuzzy set theory for monitoring processes with attribute data and at the end some guideline for the next study are proposed.
\end{abstract}

Keyword: Attribute data; Control chart; Fuzzy set theory; Statistical process control 